\title{
Dysphagia aortica dual aortic impressions on barium swallow
}

\section{M van der Ness MB ChB}

L W Stassen DMRD (Lond)

Department of Diagnostic Radiology University of the Free State Bloemfontein

\section{Abstract}

Transient intermittent oesophageal obstruction in an elderly patient is described. Barium swallow showed an extrinsic impression of the oesophagus by an aneurysmal aortic arch as well as the descending aorta. The aetiology and likely differential considerations are discussed.

\section{Introduction}

Aneurysms of the aortic arch and descending aorta can cause considerable localised displacement of the oesophagus. Dysphagia due to all types of thoracic aortic aneurysm is uncommon but well documented. The incidence has been reported to approximate $5 \%{ }^{1}$ We present a patient with two separate impressions on either side of the oesophagus due to a thoracic aortic dilatation.

\section{Case report}

An 84-year-old woman on treatment for hypertension, ischaemic heart disease and osteoporosis, presented with a 6-month history of dysphagia and weight loss. On examination the patient had kyphosis and a pulsatile epigastric mass. A barium swallow clearly demonstrated a leftsided extrinsic impression, which was due to aneurysmal dilatation of the aortic arch. A second right-sided extrinsic impression of the lower oesophagus was also demonstrated (Figs la and $1 \mathrm{~b}$ ). The impression was pulsatile and was caused by the descending aorta.

\section{Discussion}

The aorta is attached to the oesophagus by fibrous tissue. Elongation and unfolding of the ageing-descending aorta is accompanied by displacement of the oesophagus from its usual course. Dysphagia aortica can be caused by compression of either the upper oesophagus by a thoracic aneurysm or the lower oesophagus by an aneurysmal or atherosclerotic aorta. In our patient both these impressions could be demonstrated. Dysphagia aortica is associated with hypertension, old age, and kyphoscoliosis. ${ }^{2}$ Only 10 cases of dysphagia aortica were identified from a Medline search of the last 10 years and a litera-

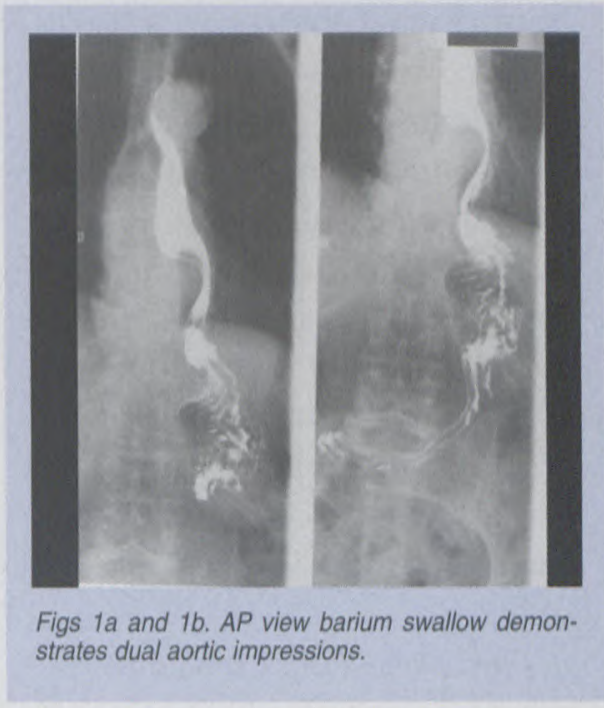

ture review compared with 40 cases of compression by an aberrant subclavian artery. ${ }^{3}$ Fluoroscopy of the barium-filled oesophagus shows transmitted pulsations. The distal oesophagus is narrowed in one plane by this extrinsic compression, and obstruction in the erect and supine positions may be relieved by turning the patient prone. Complete occlusion of the distal oesophagus is a rare manifestation of a saccular thoracic aortic aneurysm. ${ }^{4}$

The barium swallow will show a classic feature of achalasia, that is a dilated atonic oesophagus with a narrow tapered point at the cardia. Manometry, which differentiates dysphagia aortica from achalasia, shows low amplitude propagated peristaltic waves in the proximal part of the oesophagus and a high-pressure band at the site of the vascular compression. This contrasts with true achalasia in which there are no propagated contractions and no superimposed pulsations.

It is important to remember that diffuse infiltrating adenocarcinomas of the gastro-oesophageal junction can mimic the radiological and manometric features of true achalasia 
by mechanical obstruction of the distal oesophagus, as well as infiltration and destruction of the myenteric plexus by the tumour. ${ }^{5}$ This condition, termed pseudoachalasia, must be excluded before making a diagnosis of either classic achalasia or dysphagia aortica. There is no single test for pseudoachalasia, but high resolution CT, MRI, endoscopic ultrasound and careful endoscopic biopsy specimens from the area can be used to make the diagnosis.

Dysphagia aortica should be considered in any elderly patient with dysphagia who also has an aortic aneurysm. The radiographic appearance, however, needs to be distinguished from achalasia or an obstructing distal oesophageal neoplasm.

\section{References}

1. De Bakey ME, McCollum $\mathrm{CH}$, Graham JM. Surgical treatment of anuerysm of the descending thoracic aorta. J Cardiovasc Surg 1978; 19: 571-576.

2. McMillian IKR, Hyde I. Compression of oesophagus by aorta. Thorax 1969; 24: 32-38.

3. Taylor CW, Sinha A, Nightingale JMD. Dysphagia and thoracoabdominal aneurysm. Postgrad Med J 2001; 77: 257-258.

4. Mayer RC, Frank E. Saccular aortic aneurysm causing complete distal esophageal obstruction. South Med J 1993; 86: 1408-1410.

5. Robertson CS, Griffith CDM, Atkinson M, Hardcastle JD. Pseudoachalasia of the cardia: a review. JR Soc Med 1988; 81: 399-402.

\section{CT of the Head and Spine}

Nowinski

This book provides the essential information needed for formulating findings in CT of the head and spine. The book is conceived as a highly practical guide for use in routine CT diagnosis, as well as in critical oncall emergency situations.

Features:

- Condenses information to the core questions of the diagnostic problem at hand without oversimplifying: What does the clinician want to know? What must be included in the findings, and what differential diagnosis must be excluded?

- Clear and didactic organization of information in pathogenesis, clinical findings, and CT morphology

- Convenient summaries, offset by a second color, provide information to be used during the CT examination that assure that nothing will be overlooked

- Detailed descriptions of normal anatomy with normal values help to differentiate pathologic from normal findings.

2002, 426 pp, 21 tables, 420 illus., hardcover, R1 499

\section{Differential Diagnosis in Magnetic Resonance Imaging}

Burgener/Meyers/Tan

Organised by findings to reflect how radiologists really work, this abundantly illustrated book offers more than 2000 magnetic resonance images depicting commonly seen congenital and acquired disorders, as well as many rare and unusual cases. Along with the radiographic findings, you will enjoy brief tabular summaries of essential demographic,

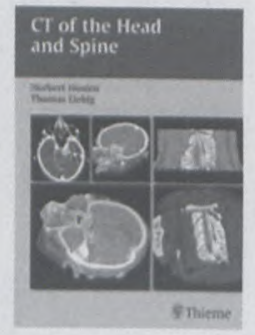
pathologic, and clinical features of each disease. The book is divided into anatomical sections, including: the brain; head and neck; spine; musculoskeletal system; chest; abdomen; and pelvis. All diseases and findings are cross-referenced, providing quick access to desired information. This is the quintessential guide to magnetic resonance imaging that radiologists and other physicians need to enhance their diagnostic skills. Keep this practical text close at hand. 2002, 704 pp, 2070 illus., hardcover, R2 375

\section{Peripheral Musculoskeletal Ultrasound Atlas}

Dondelinger / Daenen / Ferrara

Straightforward commentary and 750 illustrations - including sonograms and line drawings combine to make this book an authoritative review of high-definition ultrasonography in diagnosing musculoskeletal pathology of the extremities. This innovative, applications-oriented guide systematically covers:

- State-of-the-art instrumentation and examination techniques, with expert advice on probe positioning

- Common technical problems, diagnostic pitfalls, useful preventive and corrective actions

- Normal and pathologic ultrasound findings for muscle, tendon, ligament, periosteum and bone, joint capsule, bursa and synovium, cartilage, vessel, nerves, fat, and skin

- Pathologic regional ultrasound findings for the shoulder, arm, elbow, forearm, wrist, hand, hip, thigh, knee, leg, ankle, and foot

- A wide range of specific diagnostic applications, including diagnosis of tendon tears, hematomas, fractures, joint effusions, foreign bodies, and more.

1996, 213 pp, 750 illustrations, hardcover, R1 550

ORDERS: The SA Medical Association, Private Bag X1, Pinelands 7430.

Tel (021) 530-6527. Fax (021) 531-4126. E-mail: fpalm@samedical.org Please allow 3-4 weeks for delivery. Prices subject to foreign exchange fluctuations. 